\title{
ASYMPTOTIC SOLUTIONS TO FUCHSIAN EQUATIONS IN SEVERAL VARIABLES
}

\author{
BORIS STERNIN and VICTOR SHATALOV \\ Department of Computational Mathematics and Cybernetics, Moscow State University \\ 119899 Moscow, Russia \\ E-mail: boris@sternin.msk.su
}

\begin{abstract}
The aim of this paper is to construct asymptotic solutions to multidimensional Fuchsian equations near points of their degeneracy. Such construction is based on the theory of resurgent functions of several complex variables worked out by the authors in [1]. This theory allows us to construct explicit resurgent solutions to Fuchsian equations and also to investigate evolution equations (Cauchy problems) with operators of Fuchsian type in their right-hand parts.
\end{abstract}

1. Introduction. In this paper we construct asymptotic solutions to equations of Fuchsian type in several variables. By equations of Fuchsian type we mean equations of the form

$$
\widehat{H} u:=H\left(x, x \frac{\partial}{\partial x}\right) u=0
$$

where $x=\left(x^{1}, \ldots, x^{n}\right)$ is a point in the Cartesian space $\mathbb{R}^{n}$,

$$
x \frac{\partial}{\partial x}=\left(x^{1} \frac{\partial}{\partial x^{1}}, \ldots, x^{n} \frac{\partial}{\partial x^{n}}\right)
$$

and the function $H(x, p)$ is a polynomial with respect to the variable $p$. Such operators were studied earlier from different points of view by M. Kashiwara and T. Kawai [2], R. Melrose [3], B.-W. Schulze [4] and B. Ziemian [5], [6].

Evidently, this equation is degenerate on the union of the coordinate planes $\left\{x^{i}=0\right\}$ and, hence, one can expect that the solution will have singularities on this union. Our goal is to construct asymptotic solutions to such an equation at points of its singularities.

We remark that the set of singularities, that is, the union of the hyperplanes $\left\{x^{i}=0\right\}$ can be stratifed in such a way that the strata are coordinate planes of different dimensions. Renumbering the coordinates, if necessary, one can write down the equation of each

1991 Mathematics Subject Classification: 35A20, 35A22, 35B40, 35B65.

The paper is in final form and no version of it will be published elsewhere. 
stratum in the form

$$
A_{k}=\left\{x^{1}=0, \ldots, x^{k}=0\right\}
$$

where $k$ is the codimension of the stratum $A_{k}$.

If we intend to construct an asymptotic solution (with respect to smoothness) to equation (1) in a neighbourhood of a point of (2) then we see that the group of variables $\left(x^{1}, \ldots, x^{k}\right)$ plays quite a different role than the group $\left(x^{k+1}, \ldots, x^{n}\right)$. Indeed, the variables of the first group are transversal to the singularity manifolds $\left\{x^{i}=0, i=1, \ldots, k\right\}$ which pass through the considered point and, hence, these variables are parameters of the asymptotic expansion under construction. At the same time, the variables of the second group are not small near the considered point and can be considered simply as parameters. To stress this difference explicitly we shall slightly change the notation denoting the variables of the first group by $x=\left(x^{1}, \ldots, x^{n}\right)$ and the variables of the second one by $y=\left(y^{1}, \ldots, y^{k}\right)$. Using this notation we can rewrite equation (1) in the form

$$
\widehat{H} u=H\left(x, y, x \frac{\partial}{\partial x}, \frac{\partial}{\partial y}\right) u=0 .
$$

Further, for technical reasons it is convenient to consider the operator $\widehat{H}$ of (3) as a differential operator of the form

$$
H\left(x, x \frac{\partial}{\partial x}\right)
$$

whose coefficients lie in the space of differential operators in variables $y$ ranging over $\mathbb{R}^{n}$ or, more generally, over some smooth manifold. It is also convenient to complexify the problem with respect to the variables $x$.

For constructing asymptotic solutions to the equation (3) we use the theory of resurgent functions of several independent variables worked out by the authors (see [1]). We also remark that the one-dimensional case of that construction was considered in the paper [7] by B.-W. Schulze, B. Sternin, and V. Shatalov.

The outline of the paper is as follows. In Section 2 we construct asymptotic expansions of resurgent type for solutions to equation (3). In Section 3 we consider the corresponding evolution equations. Finally, in Section 4 we present two concrete examples of the introduced technique.

2. Statement of the problem. Let us proceed with exact definitions. Consider a Fuchsian equation of the form

$$
\widehat{H} u:=H\left(x, x \frac{\partial}{\partial x}\right) u(x)=f(x)
$$

where $\widehat{H}$ is a differential operator of the form

$$
\widehat{H}=\sum_{|\alpha| \leq m} a_{\alpha}(x)\left(x \frac{\partial}{\partial x}\right)^{\alpha}
$$

with analytic coefficients $a_{\alpha}(x)$. Here $x=\left(x^{1}, \ldots, x^{n}\right)$ is a point of the Cartesian complex 
space $\mathbb{C}^{n}$

$$
x \frac{\partial}{\partial x}=\left(x^{1} \frac{\partial}{\partial x^{1}}, \ldots, x^{n} \frac{\partial}{\partial x^{n}}\right)
$$

and we construct asymptotic solutions to equation (4) with respect to smoothness in a neighbourhood of the origin. The coefficients $a_{\alpha}(x)$ of the operator (5) can be operatorvalued functions of $x$ with values in the space of differential operators in $\mathbb{C}_{y}^{k}, y=$ $\left(y^{1}, \ldots, y^{k}\right)$, or, more generally, in the space of differential operators on a smooth manifold $Y$; in the last case we shall denote by $y$ local coordinates on $Y$.

Our approach is based on the theory of resurgent functions of several independent variables worked out in [1]. This approach gives explicit formulas for the asymptotic solutions under consideration.

Under the above asumptions equation (4) can be written in the form

$$
\widehat{H} u:=H\left(x, y, x \frac{\partial}{\partial x}, \frac{\partial}{\partial y}\right) u(x, y)=f(x, y)
$$

where

$$
H\left(x, y, x \frac{\partial}{\partial x}, \frac{\partial}{\partial y}\right)=\sum_{|\alpha| \leq m} \sum_{|\gamma| \leq m_{\alpha}} a_{\alpha}^{\gamma}(x, y)\left(x \frac{\partial}{\partial x}\right)^{\alpha}\left(\frac{\partial}{\partial y}\right)^{\gamma} .
$$

Evidently, solutions to equations (4) or (6) can have singularities on the union of the coordinate hyperplanes

$$
x^{i}=0, \quad i=1, \ldots, n .
$$

In order to apply the theory of multidimensional resurgent functions to equations (4) or (6) we perform the change of variables

$$
x^{i}=e^{\tau^{i}}, \quad i=1, \ldots, n,
$$

and expand the coefficients $a_{\alpha}(x)$ in the Taylor series in $x$ :

$$
a_{\alpha}(x)=\sum_{|\beta| \geq 0} a_{\alpha \beta} x^{\beta}=\sum_{|\beta| \geq 0} a_{\alpha \beta} e^{\tau \beta},
$$

where

$$
\tau \beta=\tau^{1} \beta_{1}+\ldots+\tau^{n} \beta_{n}
$$

and $a_{\alpha \beta}$ are differential operators in $\mathbb{C}^{n}$. Then the considered equation becomes

$$
\sum_{|\alpha| \leq m} \sum_{|\beta| \geq 0} a_{\alpha \beta} e^{\tau \beta}\left(\frac{\partial}{\partial \tau}\right)^{\alpha} u(\tau)=f\left(e^{\tau}\right)=: g(\tau) .
$$

This equation can be investigated with the help of the theory of resurgent functions of several variables presented in [1].

3. Construction of resurgent solutions. Now we are able to apply the resurgent functions theory to the construction of asymptotic solutions to equation (8). We recall [1] that a resurgent function of variables $x$ is a function of the form

$$
u(\tau)=l(U, \Omega):=\sum_{j} \int_{\Gamma_{j}} e^{-s} U(s, \tau) d s
$$


where $U(s, \tau)$ is an analytic homogeneous hyperfunction (see [8]) of the variables $(s, \tau)$ and each $\Gamma_{j}$ is a special contour surrounding some singular point $s_{j}=s_{j}(\tau)$ of the hyperfunction $U$. It is not needed that each singular point of the function $U$ is surrounded by some contour $\Gamma_{j}$; the set of singular points included in (9) is called the support of the resurgent function $u$ (see [1]) and denoted by $\Omega=\Omega(\tau)$; we emphasize that it can depend on $\tau$. The contours $\Gamma_{j}$ are shown in Figure 1 .

THEOREM 1. We have the following commutation formulas:

$$
\frac{\partial}{\partial \tau^{j}} \circ l=l \circ\left[\left(\frac{\partial}{\partial s}\right)^{-1} \frac{\partial}{\partial \tau^{j}}\right], \quad \frac{\partial}{\partial y^{j}} \circ l=l \circ \frac{\partial}{\partial y^{j}} .
$$

Further, $e^{\tau \beta} \circ l=l \circ T_{\tau \beta}$, where $T_{\tau \beta}$ is the shift operator along the $s$ axis:

$$
\left(T_{\tau \beta} U\right)(s, \tau)=U(s+\tau \beta, \tau) .
$$

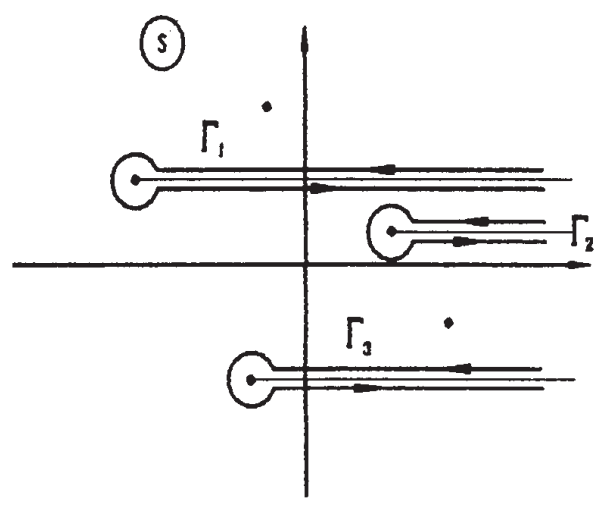

Fig. 1

Now we shall construct resurgent solutions to equation (8) provided that its right-hand side is a resurgent function. Applying Theorem 1 to equation (8) we obtain an equation for the function $U(s, \tau)$ :

$$
\sum_{|\alpha| \leq m|\beta| \geq 0} \sum_{\alpha \beta} T_{\tau \beta}\left[\left(\frac{\partial}{\partial s}\right)^{-1} \frac{\partial}{\partial \tau}\right]^{\alpha} U(s, \tau)=G(s, \tau)
$$

where $G(s, \tau)$ corresponds to $g(\tau)$ under the action of the operator $l$. Equation (10) is considered as an equation in homogeneous hyperfunctions of $s$.

We remark that, since we search for asymptotic solutions to equation (5) in a neighbourhood of the origin, the variables

$$
\tau^{j}=\ln x^{j}, \quad j=1, \ldots, n,
$$

vary in the region $\operatorname{Re} \tau^{j}<0$. Hence, we must construct asymptotic solutions with respect to smoothness to equation (10) only in this region. We recall that the coefficients $a_{\alpha \beta}$ of equation (10) are in general differential operators in $y \in \mathbb{C}^{k}$. 
Note that if $s=S(\tau)$ is a singular point of $U(s, \tau)$ then the shifted function $T_{\tau \beta}[U(s, \tau)]$ has a singularity at the point

$$
s=S(\tau)-\tau \beta
$$

lying to the right of the point of the original singularity.

Suppose that for some value of $\tau$ the point $s=S_{0}(\tau)$ is the leftmost point of the support of the resurgent function $u$ (such a point will be referred as the main singularity of the function $U$ ). Then the supports of all terms of the left-hand side of (10) except that corresponding to $\beta=0$ lie strictly to the right of the main singularity.

This allows us to use a recurrent procedure for constructing a resurgent solution to (10). Namely, we denote by $U^{0}(s, \tau)$ a solution to the equation

$$
\sum_{|\alpha| \leq m} a_{\alpha 0}\left[\left(\frac{\partial}{\partial s}\right)^{-1} \frac{\partial}{\partial \tau}\right]^{\alpha} U^{0}(s, \tau)=G(s, \tau)
$$

which is the "principal part" of (10). Then we determine the subsequent functions $U^{\beta}(s, \tau)$ as solutions to

$$
\begin{aligned}
\sum_{|\alpha| \leq m} a_{\alpha 0}\left[\left(\frac{\partial}{\partial s}\right)^{-1} \frac{\partial}{\partial \tau}\right]^{\alpha} & U^{\beta}(s, \tau) \\
& =-\sum_{|\alpha| \leq m} \sum_{\beta^{\prime}+\beta^{\prime \prime}=\beta} a_{\alpha \beta^{\prime}} T_{\tau \beta^{\prime}}\left[\left(\frac{\partial}{\partial s}\right)^{-1} \frac{\partial}{\partial \tau}\right]^{\alpha} U^{\beta^{\prime \prime}}(s, \tau)
\end{aligned}
$$

where the sum on the right does not contain the term $\beta^{\prime}=0$. Ordering the set of functions

$$
\left\{U^{\beta}(s, \tau): \beta \geq 0\right\}
$$

in such a way that the product $\tau \beta$ decreases along this ordering, we see that the system (12), (13) determines a recurrent procedure for the set of functions (14) with the same principal part.

Now we denote by $u^{\beta}(\tau)$ the resurgent function corresponding to $U^{\beta}(s, \tau)$. Certainly, we must determine the supports of these resurgent functions. To begin with, we determine these supports in a neighbourhood of some fixed value of $\tau$. The support of $U^{0}(s, \tau)$ can be chosen arbitrarily provided it is contained in a sector of angle less than $\pi$ bisected by the positive direction of the real axis in the complex $s$ plane. The supports of $u^{\beta}(\tau)$, $\beta \geq 0$, are chosen in such a way that these functions satisfy the equations

$$
\sum_{|\alpha| \leq m} a_{\alpha 0}\left(\frac{\partial}{\partial \tau}\right)^{\alpha} u^{\beta}(\tau)=-\sum_{|\alpha| \leq m} \sum_{\beta^{\prime}+\beta^{\prime \prime}=\beta} a_{\alpha \beta^{\prime}} e^{\tau \beta^{\prime}}\left(\frac{\partial}{\partial \tau}\right)^{\alpha} u^{\beta^{\prime \prime}}(\tau) .
$$

Evidently, this requirement uniquely determines the supports of $u^{\beta}(\tau)$.

In order to determine the support of $u^{\beta}(\tau)$ for all values of $\tau$ one performs analytic continuation of the constructed resurgent function along paths in the complex plane $\mathbb{C}_{s}$. This can be done in a way usual in the theory of resurgent functions with the help of the so-called transition homomorphism (see, for example, [9]). We shall not describe this construction in detail here. 
We remark that if the functions $u^{\beta}(\tau)$ are determined as described above, the series

$$
u(\tau)=\sum_{\beta \geq 0} u^{\beta}(\tau)
$$

converges in the space of resurgent functions since we consider the domain in the space $\mathbb{C}_{\tau}^{n}$ where $\operatorname{Re} \tau \beta \leq 0$ and, hence, the supports of the terms of this series lie in the half-plane $\operatorname{Re} s>N$ for any value of $N$ if $|\beta|$ is sufficiently large. The function (15) is exactly the required resurgent solution to $(8)$.

Note that since we search for a resurgent solution of the initial equation, we must solve equation (12) for the microfunction $U^{0}$ (as well as the subsequent equations for microfunctions $U^{\beta}$ ) in the class of infinitely continuable microfunctions. To investigate the existence of such solutions we use the $\partial / \partial s$-transformation of ramifying analytic functions (see [10]). Applying this transformation to equation (12) we obtain the following equation for the image $\widetilde{U}^{0}(s, p)$ of the function $U^{0}(s, \tau)$ under this transformation:

$$
\sum_{|\alpha| \leq m} a_{\alpha 0} p^{\alpha} \widetilde{U}^{0}(s, p)=\widetilde{G}(s, p) .
$$

The latter equation is a family of operator equations in the space of functions of $y$ with parameters $p \in \mathbb{C}_{n}$. Note that the latter equation must be solved in the space of microfunctions, that is, we must solve equation (16) modulo holomorphic functions of $(s, p)$. Similar to the case of differential equations with constant (numerical) coefficients, the set of singularities of the solution $\widetilde{U}^{0}(s, p)$ is determined by the set of points $p \in \mathbb{C}_{n}$ such that the operator

$$
\widetilde{H}(p)=\sum_{|\alpha| \leq m} a_{\alpha 0} p^{\alpha}
$$

is not invertible in the considered space of functions of $y$. We denote this set by

$$
\text { char } \widehat{H}=\{p: \widetilde{H}(p) \text { is not invertible }\}
$$

and call it the characteristic set of the operator $\widehat{H}$. We impose the following requirement on $\widehat{H}$ :

Condition 1. char $\widehat{H}$ is an analytic set in $\mathbb{C}_{n}$.

Under this condition the set of singularities of a solution to equation (12) is the union of some set which is characteristic with respect to $\widehat{H}$ and the set of singularities of the right-hand side $G(s, \tau)$. Suppose that the main singularity $s=S_{0}(\tau)$ of the solution is not determined by any singularity of the function $G(s, \tau)$. Then $S_{0}(\tau)$ must be a solution of the Hamilton-Jacobi equation

$$
\left\{p: p=\partial S_{0} / \partial \tau\right\} \subset \operatorname{char} \widehat{H} .
$$

Now we are able to prove the existence of infinitely continuable solutions to equation (12).

THEOREM 2. Suppose that the operator $\widehat{H}$ satisfies Condition 1. Then equation (12) is solvable in the space of resurgent functions.

P r o of. To construct a solution to equation (12) we choose a submanifold which is not everywhere characteristic with respect to this equation. Then a solution to any Cauchy 
problem with resurgent Cauchy data on this manifold will be a resurgent solution to equation (12). The existence of an infinitely continuable solution for such a problem (under the condition that the Cauchy data are infinitely continuable) can be proved with the help of an explicit formula for solutions which has the same form as in the case of constant (numerical) coefficients (see [10]). The proof of the fact that this formula determines an infinitely continuable solution to the Cauchy problem is quite similar to that in the cited book and we leave it to the reader. This proves the theorem.

To conclude this section, we present the form of constructed asymptotic solutions in the case when this solution has simple singularities. We recall that the resurgent function (9) has simple singularities if the corresponding function $U(s, \tau)$ can be represented in a neighbourhood of its singular points in the form

$$
U(s, \tau)=\frac{a_{0}(\tau)}{s-S(\tau)}+\ln (s-S(\tau)) \sum_{j=0}^{\infty} \frac{(s-S(\tau))^{j}}{j !} a_{j+1}(\tau)
$$

where $s=S(\tau)$ is an equation of the singularity set of $U$ and the series on the right converges in a neighbourhood of $s=S(\tau)$. From the homogeneity properties of $U$ it follows that $S(\tau)$ is a homogeneous function of $\tau$ of degree 1 and $a_{j+1}(\tau)$ are homogeneous functions of order $-(j+1)$. It is known that if $u(\tau)$ is a resurgent function with simple singularities then the point $s=S(\tau)$ of singularity corresponds to the term

$$
e^{-S(\tau)} \sum_{j=0}^{\infty} a_{j}(\tau)
$$

of the asymptotic expansion for large values of $|\tau|$. Performing the change of variables (11) we come to the asymptotic expansion of the initial function $u(x)$ which is the sum of the following terms:

$$
e^{-S(\ln x)} \sum_{j=0}^{\infty} a_{j}(\ln x)
$$

where $S$ and $a_{j}$ are homogeneous functions of degree 1 and $-j$ respectively.

4. Evolution equations. In this section we consider the Cauchy problem

$$
\left\{\begin{array}{l}
\frac{\partial^{m} u}{\partial t^{m}}=\widehat{H} u \\
\left.u\right|_{t=0}=u_{0}(x), \ldots,\left.\frac{\partial^{m-1} u}{\partial t^{m-1}}\right|_{t=0}=u_{m-1}(x),
\end{array}\right.
$$

where $\widehat{H}$ is an operator of the type (5). As above, using an exponential change of variables (7) and expanding the coefficients of $\widehat{H}$ in Taylor series in $x$, we reduce $(20)$ to the form

$$
\left\{\begin{array}{l}
\frac{\partial^{m} u}{\partial t^{m}}=\sum_{|\alpha| \leq m} \sum_{|\beta| \geq 0} a_{\alpha \beta} e^{\tau \beta}\left(\frac{\partial}{\partial \tau}\right)^{\alpha} u \\
\left.u\right|_{t=0}=u_{0}(\tau), \ldots,\left.\frac{\partial^{m-1} u}{\partial t^{m-1}}\right|_{t=0}=u_{m-1}(\tau) .
\end{array}\right.
$$

Remark 1. We recall that $a_{\alpha \beta}$ in the latter equation are supposed to be differential operators in $y \in \mathbb{C}^{k}$. More generally, we can assume that these operators contain 
differentiations with respect to $t$ of order not more than $m-1$. In any case, the orders of the operators $a_{\alpha \beta}$ are supposed to be less than or equal to $m-|\alpha|$.

Similar to the previous section we search for a solution to problem (21) in the form of a resurgent function (see equation (9)):

$$
u(t, \tau)=\sum_{j} \int_{\Gamma_{j}} e^{-s} U(s, t, \tau) d s,
$$

where $U(s, t, \tau)$ is an infinitely continuable analytic function in $s$. The corresponding Cauchy problem for $U(s, t, \tau)$ has the form

$$
\left\{\begin{array}{l}
\frac{\partial^{m} U}{\partial t^{m}}=\sum_{|\alpha| \leq m} \sum_{|\beta| \geq 0} a_{\alpha \beta} T_{\tau \beta}\left[\left(\frac{\partial}{\partial s}\right)^{-1}\left(\frac{\partial}{\partial \tau}\right)\right]^{\alpha} U \\
\left.U\right|_{t=0}=U_{0}(s, \tau), \ldots,\left.\frac{\partial^{m-1} U}{\partial t^{m-1}}\right|_{t=0}=U_{m-1}(s, \tau) .
\end{array}\right.
$$

We shall construct a solution to (22) with the help of a recurrent procedure. Namely, we define $U^{0}(s, t, \tau)$ as a solution of the Cauchy problem

$$
\left\{\begin{array}{l}
\frac{\partial^{m} U^{0}}{\partial t^{m}}=\sum_{|\alpha| \leq m} a_{\alpha 0}\left[\left(\frac{\partial}{\partial s}\right)^{-1}\left(\frac{\partial}{\partial \tau}\right)\right]^{\alpha} U^{0} \\
\left.U^{0}\right|_{t=0}=U_{0}(s, \tau), \ldots,\left.\frac{\partial^{m-1} U^{0}}{\partial t^{m-1}}\right|_{t=0}=U_{m-1}(s, \tau)
\end{array}\right.
$$

Then, for each multiindex $\beta \neq 0$ we determine $U^{\beta}(s, t, \tau)$ as a solution to the Cauchy problem

$$
\left\{\begin{array}{l}
\frac{\partial^{m} U^{\beta}}{\partial t^{m}}=\sum_{|\alpha| \leq m} a_{\alpha 0}\left[\left(\frac{\partial}{\partial s}\right)^{-1}\left(\frac{\partial}{\partial \tau}\right)\right]^{\alpha} U^{\beta} \\
+\sum_{|\alpha| \geq m} \sum_{\beta^{\prime}+\beta^{\prime \prime}=\beta} a_{\alpha \beta^{\prime}} T_{\tau \beta^{\prime}}\left[\left(\frac{\partial}{\partial s}\right)^{-1}\left(\frac{\partial}{\partial \tau}\right)\right]^{\alpha} U^{\beta^{\prime \prime}} \\
\left.U^{\beta}\right|_{t=0}=0, \ldots,\left.\frac{\partial^{m-1} U^{\beta}}{\partial t^{m-1}}\right|_{t=0}=0
\end{array}\right.
$$

where the last sum does not contain the term with $\beta^{\prime}=0$. Since $\operatorname{Re}(\tau \beta) \leq 0$, the second term on the right-hand side of the equation in (24) contains the functions $U^{\beta^{\prime \prime}}$ only with $\operatorname{Re}\left(\tau \beta^{\prime \prime}\right)>\operatorname{Re}(\tau \beta)$. Hence, if we order the set of functions $\left\{U^{\beta}: \beta \geq 0\right\}$ in such a way that $\operatorname{Re}(\tau \beta)$ does not increase, the set of Cauchy problems determines a recurrent procedure for determining these functions. Certainly, for problems (23), (24) to be solvable in the class of infinitely continuable functions, one has to impose some requirements on the operator on the right-hand side of (23); such requirements will be imposed below. However, if we assume that the recurrent system $(23),(24)$ is solvable in the required function class, then the series

$$
u(t, \tau)=\sum_{\beta \geq 0} u^{\beta}(t, \tau)
$$


converges in the space of resurgent functions (here $u^{\beta}(t, \tau)$ are resurgent functions corresponding to $\left.U^{\beta}(s, t, \tau)\right)$ since the supports of $u^{\beta}(t, \tau)$ move to the left along the described ordering. The resurgent function (25) evidently is a resurgent solution for the problem (21).

Now let us formulate the condition under which equation (23) is solvable in classes of infinitely continuable functions. To do this, we apply the $\partial / \partial s$-transformation [10] to the problem (23). Setting

$$
\widetilde{U}^{\beta}(s, t, \tau)=F_{\partial / \partial s}\left(U^{\beta}(s, t, \tau)\right)
$$

we come to the following Cauchy problems:

$$
\begin{aligned}
& \left\{\begin{array}{l}
\frac{\partial^{m} \widetilde{U}^{0}}{\partial t^{m}}=\sum_{|\alpha| \leq m} a_{\alpha 0} p^{\alpha} \widetilde{U}^{0}, \\
\left.\widetilde{U}^{0}\right|_{t=0}=\widetilde{U}_{0}(s, p), \ldots,\left.\frac{\partial^{m-1} \widetilde{U}^{0}}{\partial t^{m-1}}\right|_{t=0}=\widetilde{U}_{m-1}(s, p),
\end{array}\right. \\
& \left\{\begin{array}{l}
\frac{\partial^{m} \widetilde{U}^{\beta}}{\partial t^{m}}=\sum_{|\alpha| \leq m} a_{\alpha 0} p^{\alpha} U^{\widetilde{\beta}}+\sum_{|\alpha| \leq m} \sum_{\beta^{\prime}+\beta^{\prime \prime}=\beta} a_{\alpha \beta^{\prime}} T_{\tau \beta^{\prime}} p^{\alpha} \widetilde{U}^{\beta^{\prime \prime}}, \\
\left.\widetilde{U}^{\beta}\right|_{t=0}=0, \ldots,\left.\frac{\partial^{m-1} \widetilde{U}^{\beta}}{\partial t^{m-1}}\right|_{t=0}=0
\end{array}\right.
\end{aligned}
$$

for the functions (26). Evidently, the solvability of (23) is equivalent to the solvability of (27), so we must impose the following condition.

Condition 2. The solution operator for problem (27) exists for each value of $p \in \mathbb{C}_{n}$ and determines an analytic family of operators with parameter $p$.

Let us describe a situation in which Condition 2 will be valid. Suppose that the order of the operator $\left(^{1}\right) a_{\alpha 0}$ equals $m-|\alpha|$ and that the operator

$$
\frac{\partial^{m}}{\partial t^{m}}-a_{00}
$$

is strictly hyperbolic (see Remark 1 above). Then it is evident that Condition 2 is valid.

To conclude this section we investigate the singularities of $U^{\beta}(s, t, \tau)$ provided that the Cauchy data $U_{j}(s, \tau)$ of problem (23) have simple singularities. This means that $U_{j}(s, \tau), j=1, \ldots, m-1$, can be represented in the form

$$
U_{j}(s, \tau)=\frac{a_{0}(\tau)}{s-S(\tau)}+\ln (s-S(\tau)) \sum_{i=0}^{\infty} \frac{(s-S(\tau))^{i}}{i !} a_{i+1}(\tau)
$$

near each point $s=S(\tau)$, where the series on the right converges. Then, as follows from the stationary phase formula for the $\partial / \partial s$-transformation (see [10], [11], [12]), the functions $\widehat{U}_{j}(s, p)$ have the same form

$$
\widetilde{U}_{j}(s, p)=\frac{\widetilde{a}_{0}(p)}{s-\widetilde{S}(p)}+\ln (s-\widetilde{S}(p)) \sum_{i=0}^{\infty} \frac{(s-\widetilde{S}(p))^{i}}{i !} \widetilde{a}_{i+1}(p)
$$

$\left.{ }^{1}\right)$ We recall that $a_{\alpha \beta}$ are supposed to be differential operators in variables $y$. 
near singular points $s=\widetilde{S}(p)$, where $\widetilde{S}(p)$ is the Legendre transform of $S(\tau)$. Now the singularities of $\widetilde{U}^{\beta}(s, t, p)$ can be computed in the usual way with the help of the Hamiltonian flow along the trajectories of the operator appearing in problems (27) and (28). Note that, in contrast to the case considered in Section 3 the operator itself does not originate any singularities of solution; all singularities come from the singularities of the Cauchy data.

5. Examples. In this section we consider two examples of constructing a resurgent solution to a stationary equation and to a Cauchy problem.

EXAmple 1. Consider the equation

$$
\left[\left(x^{1} \frac{\partial}{\partial x^{1}}\right)^{2}+\left(x^{2} \frac{\partial}{\partial x^{2}}\right)^{2}+\frac{\partial^{2}}{\partial y^{2}}\right] u\left(x^{1}, x^{2}, y\right)=0,
$$

where $x^{1}$ and $x^{2}$ are in a neighbourhood of the origin in $\mathbb{C}^{2}$ and $y$ belongs to the unit circle $S^{1}$. The corresponding characteristic set (17) for this equation is the union of the sets

$$
\operatorname{char}_{n} \widehat{H}=\left\{p_{1}^{2}+p_{2}^{2}-n^{2}=0\right\}
$$

over all natural numbers $n$. This follows from the fact that the operator

$$
\frac{\partial^{2}}{\partial y^{2}}+p_{1}^{2}+p_{2}^{2}
$$

on the unit circle is not invertible exactly for values of $p=\left(p_{1}, p_{2}\right)$ such that $p_{1}^{2}+p_{2}^{2}=n^{2}$. Performing, similar to the general case, the change (7) of variables and passing to the "resurgent images" $U\left(s, \tau^{1}, \tau^{2}, y\right)$ via formula (9), we come to an equation for $U$ of the form

$$
\left[\left(\frac{\partial}{\partial s}\right)^{-2}\left(\frac{\partial}{\partial \tau^{1}}\right)^{2}+\left(\frac{\partial}{\partial s}\right)^{-2}\left(\frac{\partial}{\partial \tau^{2}}\right)^{2}+\frac{\partial}{\partial y^{2}}\right] U\left(s, \tau^{1}, \tau^{2}, y\right)=0 .
$$

As follows from the considerations of Section 3 the singularities of a solution to the latter equation must lie in the set $s=S\left(\tau^{1}, \tau^{2}, y\right)$ where the function $S$ must be a solution to one of the Hamilton-Jacobi equations

$$
\left(\frac{\partial S}{\partial \tau^{1}}\right)^{2}+\left(\frac{\partial S}{\partial \tau^{2}}\right)^{2}=n^{2}
$$

for some nonnegative integer $n$. We denote a solution of this equation by $S_{n}\left(\tau^{1}, \tau^{2}\right)$.

Now we can construct an asymptotic solution to equation (30) with simple singularities. Such a solution has the form

$$
U\left(s, \tau^{1}, \tau^{2}, y\right)=U_{+}\left(s, \tau^{1}, \tau^{2}\right) e^{i n y}+U_{-}\left(s, \tau^{1}, \tau^{2}\right) e^{-i n y}
$$

where the functions $U_{ \pm}$are solutions to the equation

$$
\left[\left(\frac{\partial}{\partial s}\right)^{-2}\left(\frac{\partial}{\partial \tau^{1}}\right)^{2}+\left(\frac{\partial}{\partial s}\right)^{-2}\left(\frac{\partial}{\partial \tau^{2}}\right)^{2}-n^{2}\right] U_{ \pm}\left(s, \tau^{1}, \tau^{2}, y\right)=0 .
$$

Such form of solution is due to the fact that the functions $\exp ( \pm i n y)$ are eigenfunctions of the operator $\partial^{2} / \partial y^{2}$ on the unit circle $S^{1}$. Solutions to equation (33) of the form (29) corresponding to the action (31) can easily be constructed with the help of the Maslov 
canonical operator on complex manifolds (see [10]). We present here only the result of the computation. Computations similar to those in [13] give

$$
S_{n}=S_{n}^{ \pm}\left(\tau^{1}, \tau^{2}\right)= \pm n \sqrt{\left(\tau^{1}\right)^{2}+\left(\tau^{2}\right)^{2}}
$$

or

$$
S_{n}=S_{n}\left(\tau^{1}, \tau^{2}\right)=n\left(a \tau^{1}, b \tau^{2}\right)
$$

where $a$ and $b$ are subject to the relation $a^{2}+b^{2}=1$.

By (19) the terms $U_{ \pm}$of the asymptotic expansion (32) corresponding to the actions $S_{n}^{ \pm}\left(\tau^{1}, \tau^{2}\right)$ have the form

$$
e^{ \pm n \sqrt{\left(\ln x^{1}\right)^{2}+\left(\ln x^{2}\right)^{2}}} \sum_{j=0}^{\infty} a_{j}\left(\ln x^{1}, \ln x^{2}\right)
$$

where $a_{j}\left(\tau^{1}, \tau^{2}\right)$ are homogeneous in $\left(\tau^{1}, \tau^{2}\right)$ of degree $-j$.

Asymptotic solutions to the nonhomogeneous equation

$$
\left[\left(x^{1} \frac{\partial}{\partial x^{1}}\right)^{2}+\left(x^{2} \frac{\partial}{\partial x^{2}}\right)^{2}+\frac{\partial}{\partial y^{2}}\right] u\left(x^{1}, x^{2}, y\right)=f\left(x^{1}, x^{2}, y\right)
$$

with resurgent right-hand side $f\left(x^{1}, x^{2}, y\right)$ can be investigated with the help of the Green function of equation (33). The corresponding computations are similar to those in [7].

ExAmple 2. Consider the Cauchy problem

$$
\left\{\begin{array}{l}
\frac{\partial^{2} u}{\partial t^{2}}=\left[\left(x^{1} \frac{\partial}{\partial x^{1}}\right)^{2}+\left(x^{2} \frac{\partial}{\partial x^{2}}\right)^{2}+\frac{\partial^{2}}{\partial y^{2}}\right] u, \\
\left.u\right|_{t=0}=u_{0},\left.\quad \frac{\partial u}{\partial t}\right|_{t=0}=u_{1} .
\end{array}\right.
$$

Here, similar to the previous example, the variable $y$ varies on the unit circle $S^{1}$ and we construct asymptotic solutions in a neighbourhood of the origin in the space of the variables $x=\left(x^{1}, x^{2}\right)$. We require also that $u_{0}$ and $u_{1}$ are resurgent functions of the variables $\tau=\left(\tau^{1}, \tau^{2}\right)$ determined by the change (7) of variables. This means that the functions $u_{0}\left(e^{\tau^{1}}, e^{\tau^{2}}\right), u_{1}\left(e^{\tau^{1}}, e^{\tau^{2}}\right)$ can be represented in the form of the integral (9) with the corresponding functions $U_{0}(s, \tau, y)$ and $U_{1}(s, \tau, y)$. Then the Cauchy problem for $U=U(s, t, \tau, y)$ has the form

$$
\left\{\begin{array}{l}
\frac{\partial^{2} U}{\partial t^{2}}=\left[\left(\frac{\partial}{\partial s}\right)^{-2}\left(\frac{\partial}{\partial \tau^{1}}\right)^{2}+\left(\frac{\partial}{\partial s}\right)^{-2}\left(\frac{\partial}{\partial \tau^{2}}\right)^{2}+\frac{\partial^{2}}{\partial y^{2}}\right] U, \\
\left.U\right|_{t=0}=U_{0},\left.\quad \frac{\partial U}{\partial t}\right|_{t=0}=U_{1} .
\end{array}\right.
$$

Passing in this Cauchy problem to the image $\widetilde{U}(s, t, p, y)$ of $U(s, t, \tau, y)$ under the action of the $\partial / \partial s$-transformation we come to the following family of Cauchy problems with the parameters $p=\left(p_{1}, p_{2}\right)$ :

$$
\left\{\begin{array}{l}
\frac{\partial^{2} \widetilde{U}}{\partial t^{2}}=\left[\left(p_{1}\right)^{2}+\left(p_{2}\right)^{2}+\frac{\partial^{2}}{\partial y^{2}}\right] \widetilde{U} \\
\left.\widetilde{U}\right|_{t=0}=\widetilde{U}_{0},\left.\quad \frac{\partial \widetilde{U}}{\partial t}\right|_{t=0}=\widetilde{U}_{1}
\end{array}\right.
$$


where $\widetilde{U}_{0}$ and $\widetilde{U}_{1}$ are the images of the Cauchy data $U_{0}$ and $U_{1}$ of problem (34) under the $\partial / \partial s$-transformation.

Suppose now that the functions $\widetilde{U}_{j}, j=1,2$, have simple singularities, that is,

$$
\widetilde{U}_{j}(s, p, y)=\frac{a_{0}(p, y)}{s-S(p, y)}+\ln (s-S(p, y)) \sum_{i=0}^{\infty} \frac{(s-S(p, y))^{i}}{i !} a_{i+1}(p, y) .
$$

Suppose, in addition, that

$$
\frac{\partial S(p, y)}{\partial y} \neq 0
$$

Then, as follows from [14], the asymptotic solution to problem (34) with respect to smoothness has the form

$$
\widetilde{U}(s, t, p, y)=\widetilde{U}^{+}(s, t, p, y)+\widetilde{U}^{-}(s, t, p, y)
$$

where

$$
\widetilde{U}_{j}^{ \pm}(s, t, p, y)=\frac{a_{0}^{ \pm}(t, p, y)}{s-S^{ \pm}(t, p, y)}+\ln \left(s-S^{ \pm}(t, p, y)\right) \sum_{i=0}^{\infty} \frac{\left(s-S^{ \pm}(t, p, y)\right)^{i}}{i !} a_{i+1}^{ \pm}(t, p, y)
$$

and the functions $S^{ \pm}(t, p, y)$ are solutions of the following Cauchy problem for the Hamilton-Jacobi equation:

$$
\left\{\begin{array}{l}
\frac{\partial S^{ \pm}(t, p, y)}{\partial t}= \pm \frac{\partial S^{ \pm}(t, p, y)}{\partial y} \\
\left.S^{ \pm}(t, p, y)\right|_{t=0}=S(p, y)
\end{array}\right.
$$

The explicit asymptotics for solutions to problem (34) with respect to smoothness can be obtained with the help of the Laplace-Radon integral operators on complex manifolds; the theory of these operators is presented in the book [10].

\section{References}

[1] B. Yu. Sternin and V. E. Shatalov, On a notion of resurgent function of several variables, Math. Nachr. 171 (1995), 283-301.

[2] M. Kashiwara and T. Kawai, Second microlocalization and asymptotic expansions, in: Lecture Notes in Phys. 126, Springer, New York, 1980, 21-76.

[3] R. Melrose, Analysis on Manifolds with Corners, Lecture Notes, MIT, Cambridge, Mass., 1988.

[4] B.-W. Schulze, Pseudodifferential Operators on Manifolds with Singularities, NorthHolland, Amsterdam, 1991.

[5] B. Ziemian, Elliptic corner operators in spaces with continuous radial asymptotics. I, J. Differential Equations 101 (1993), 28-57.

[6] - Elliptic corner operators in spaces with continuous radial asymptotics. II, in: Partial Differential Equations, Banach Center Publ. 27, Inst. of Math., Polish Acad. Sci., Warszawa, 1992, 555-580.

[7] B.-W. Schulze, B. Sternin and V. Shatalov, Resurgent analysis in the theory of differential equations with singularities, Math. Nachr. 170 (1994), 1-21.

[8] H. Komatsu, Laplace transform of hyperfunctions. A new foundation of Heaviside calculus, J. Fac. Sci. Univ. Tokyo IA 34 (1987), 805-820. 
[9] B. Candelpergher, J. C. Nosmas and F. Pham, Approche de la Résurgence, Hermann, 1993.

[10] B. Yu. Sternin and V. E. Shatalov, Differential Equations on Complex Manifolds, Kluwer Acad. Publ., Dordrecht, 1994.

[11] - - - On a formula for the asymptotic expansion of an integral in complex analysis, Soviet Math. Dokl. 43 (1991), 624-627.

[12] - - - Stationary phase method for Laplace-Radon transformation, Mat. Zametki 51 (4) (1992), 116-125 (in Russian).

[13] - - - On exact asymptotics at infinity of solutions to differential equations, preprint, Max-Planck-Institut für Mathematik, Bonn, 1993.

[14] M. V. Korovina, B. Yu. Sternin and V. E. Shatalov, Asymptotical expansions "in the large" of solutions of the complex Cauchy problem with singular initial data, Soviet Math. Dokl. 44 (1991), 674-677. 